\section{0 conceito de qualidade do cliente aplicado ao serviço de alimentação e nutrição de hospital oncológico}

\section{The concept of customer quality applied to the food and nutrition service of an oncology hospital}

\author{
Janaine Salete Lorencet ${ }^{l}$ \\ Keith Selbmann ${ }^{2}$ \\ Ivone Mayumi Ikeda Morimoto ${ }^{3}$ \\ Marina Lopes ${ }^{4}$ \\ Camila Brandão Polakowski ${ }^{5}$
}

\begin{abstract}
Resumo
O Serviço de Alimentação e Nutrição hospitalar é responsável não somente por produzir e distribuir refeições, é também sua função atender às necessidades do cliente hospitalizado. $\mathrm{O}$ objetivo deste estudo foi avaliar resultados de estratégias para melhoria da qualidade do Serviço de Alimentação e Nutrição de um hospital oncológico a partir da análise de fatores determinantes da qualidade na visão deste cliente. Após aplicação de questionário para conhecer graus de importância atribuídos às características da qualidade da alimentação, foram listados objetivos, resultados esperados e ações. Houve comparação da satisfação do cliente hospitalizado antes e após a implementação das estratégias. A frequência de respostas "satisfatório" relativas à aparência das refeições, atendimento da copeira e horário de refeições aumentou. Não houve aumento do percentual de aceitação da dieta, mas o motivo de não aceitação "características organolépticas" e "quantidade exagerada de alimentos", dependentes do serviço de alimentação ofertado, apresentou redução da frequência. Dentre os motivos de não aceitação relacionados a doença, foi possível identificar prevalência sintomas consequentes ao tratamento quimioterápico, radioterápico ou cirúrgico a que o paciente oncológico é submetido como a falta de apetite e presença de distúrbios gastrointestinais nos dois momentos porém, com aumento de $73,73 \%$ para $79,29 \%$, confirmando que a aceitação alimentar recebeu grande influência de fatores relacionados à doença.

Palavras-chave: Cliente. Gastronomia hospitalar. Gestão. Qualidade. Qualidade em serviços. Serviços hospitalares. sistemas de saúde. hospital oncológico.
\end{abstract}

\begin{abstract}
The hospital Food and Nutrition Service is responsible not only for producing and distributing meals. It is your job to meet the needs of the hospitalized client. The objective of this study was to evaluate the results of strategies to improve the quality of the Food and Nutrition Service of an oncology hospital based on the analysis of factors that determine quality in the view of this client. After applying a questionnaire to find out the degrees of importance attributed to the characteristics of the quality of the food, objectives, expected results and actions were listed. There was a comparison of the satisfaction of hospitalized clients before and after the implementation of the strategies. The frequency of "satisfactory" responses related to the appearance of meals, attendance at the maid and meal times increased. There was no increase in the percentage of acceptance of the diet, but the reason for not accepting "organoleptic characteristics" and "exaggerated amount of food", depending on the food service offered, showed a reduction in frequency. Among the reasons for nonacceptance related to the disease, it was possible to identify the prevalence of symptoms resulting from chemotherapy, radiotherapy or surgical treatment to which the cancer patient is submitted, such as lack of appetite and presence of gastrointestinal disorders at both times, with an increase of $73,73 \%$ to $79.29 \%$, confirming that food acceptance received a great influence from factors related to the disease.

Keywords: Client. Hospital gastronomy. Management. Quality. quality of services. hospital services. health systems. oncology hospital.
\end{abstract}

${ }^{1}$ Pontifícia Universidade Católica do Paraná- PUCPR. E-mail: janainelorenceti@hotmail.com

${ }^{2}$ Nutricionista e Técnica em Nutrição e Dietética, formada pela Pontifícia Universidade Católica do Paraná. Pós graduanda em Nutrição Hospitalar

Clínica e Terapia Nutricional em Cuidados Intensivos pela UniAmérica. E-mail: keithselbmann@hotmail.com

${ }^{3}$ Mestre em Engenharia de Produção pela Universidade Federal de Santa Catarina. Professora e membro do Núcleo de Excelência Pedagógica da Escola de Ciências da Vida - PUCPR. E-mail: ivone.morimoto@pucpr.br

${ }^{4}$ Pós-graduada em Nutrição Clínica pelo Colégio Brasileiro de Estudos Sistêmicos. Supervisora do Serviço de Nutrição e Dietética do Hospital Erasto Gaertner. E-mail: mlopes@erastogaertner.com.br

${ }^{5}$ Mestre em Segurança Alimentar e Nutricional pela Universidade Federal do Paraná. E-mail: camilapolakowski@hotmail.com

Artigo recebido em: 12 de agosto de 2020. Artigo aceito em 14 de fevereiro de 2021. 


\section{Introdução}

O Serviço de Alimentação e Nutrição (doravante SAN) hospitalar tem como objetivo a preparação e oferta de refeições sensorialmente agradáveis, adequadas do ponto de vista nutricional e com qualidade higiênico-sanitária. Devem atender às necessidades do público alvo, promovendo a satisfação de seus usuários (ROMERO; ANGST, 2015; VIEIRA et al., 2016; BANKS et al., 2017; TRANCOSO; TOMASIAK, 2004).

Inúmeros estudos demonstram que a aceitação alimentar tem papel fundamental na manutenção do estado nutricional de pacientes hospitalizados e que a desnutrição resulta em aumento da mortalidade, da incidência de complicações e do tempo de hospitalização (AQUINO; PHILIPPI, 2011; FERREIRA; GUIMARÃES; MARCADENTI, 2013; PALMIERI et al., 2013; RIBAS et al., 2013).

Em hospitais oncológicos, a má aceitação da alimentação tem como causa a doença propriamente dita, que pode acometer órgãos que impedem a ingestão alimentar adequada, bem como os tratamentos quimioterápico, radioterápico ou cirúrgico a que o paciente é submetido para controle da doença. Estas formas de tratamento podem trazer consequências indesejáveis como alteração no paladar, falta de apetite, aversões alimentares e alterações mecânicas e funcionais do trato gastrointestinal como náuseas, vômitos, diarreia, mucosite e baixa salivação (CARVALHO; CAMILO; RAVASCO, 2011).

Além deste aspecto fisiológico, ao receber o diagnóstico de câncer, o indivíduo é cercado por "um estigma aterrorizante, pois desperta nas pessoas sentimentos como: raiva, medo, angústia, impotência, desamparo, tristeza, desespero e principalmente medo da morte"' (SALCI; MARCON, 2010). Estes," vivenciam sentimentos de medo da cirurgia, medo da quimioterapia e de seus efeitos colaterais, além da sensação de impotência diante da situação"' (FIGUEIREDO et al., 2017).

Segundo Banks et al. (2017), a qualidade do SAN influencia a aceitação alimentar e a satisfação geral do cliente, portanto, o nutricionista que gerencia o serviço de alimentação hospitalar deve considerar o conceito de qualidade do cliente. Para Juran (1992), ao conhecer o conceito de qualidade na visão do cliente, as atividades passam a ser direcionadas para o atendimento de suas expectativas, assim como o processo que 
deve ser constante e continuamente monitorado e melhorado, pois as necessidades e desejos se modificam constantemente (CARPINETTI, 2012)

Recentemente, os serviços de saúde deixaram de ser entendidos apenas como locais de promoção, prevenção e assistência aos pacientes e assumem uma postura de organização que necessita de gerenciamento com foco no cliente (SAIATO et al., 2013). "Instituições mais preparadas, com processos mais estruturados, irão certamente assegurar a esses clientes garantias de melhor assistência e, portanto, um restabelecimento de saúde com práticas mais definidas e disseminadas na instituição" (BONATO, 2011).

Cabe ressaltar que quando se refere ao indivíduo hospitalizado são utilizados os termos paciente e cliente. O termo paciente começou a ser utilizado no século XIV e tem como significado a pessoa que tem paciência, que é conformado, portanto, a sua utilização sugere uma posição passiva. Já o termo cliente, tem sua origem de um vocabulário próprio da economia liberal de mercado que reconhece a pessoa que recorre ao serviço de saúde como um consumidor, com voz ativa.

Dentro da premissa de paciente visto como cliente, o objetivo geral deste estudo foi avaliar resultados de estratégias para melhoria da qualidade do SAN de um hospital oncológico a partir dos fatores determinantes da qualidade na visão deste cliente.

\section{Método}

Trata-se de um estudo de caráter quantitativo experimental, realizado no Serviço de Alimentação e Nutrição do Hospital Oncológico Erasto Gaertner da cidade de Curitiba-PR. Este estudo foi aprovado pelo Comitê de Ética em Pesquisa do Hospital Erasto Gaertner, sob o parecer de no 3.508.294/2019. Neste estudo, o termo "paciente" foi substituído por "cliente hospitalizado".

Inicialmente foi realizada a tabulação de dados de um questionário estruturado segundo Morimoto (2002), aplicado entre os meses de março e maio de 2019 pelo serviço de nutrição do hospital. Os graus muito importantes (MI), importante (I), indiferente (IN), pouco importante (PI) e sem importância (SI) foram avaliados pelos clientes considerando os seguintes atributos: aparência visual, sabor, temperatura adequada dos alimentos, cardápio variado, higiene dos alimentos, cortesia no atendimento, aparência dos funcionários que servem as refeições, utensílios adequados para alimentar-se (embalagem, bandeja, talheres) e condições adequadas para alimentar- 


\section{LORENCET; SELBMANN; MORIMOTO; LOPES; POLAKOWSKI}

se (mobília). Foi estabelecido como determinantes da qualidade, os atributos nos quais a avaliação estatística da moda resultou em muito importante (MI) e importante (I).

Concomitantemente, foi realizada uma pesquisa de aceitação da dieta, registrando-se o percentual de ingestão da refeição almoço, segundo o relato do próprio paciente $(0 \%, 25 \%, 50 \%, 75 \%$ ou $100 \%$ da refeição) e quais seriam os motivos da não aceitação de $100 \%$ da alimentação ofertada.

Nos dois levantamentos realizados, foram incluídos clientes internados em apartamentos (atendidos por operadora de planos de saúde) e enfermarias (atendidos pelo Sistema Único de Saúde) maiores de 18 anos que estavam em condições de verbalizar para responder aos questionários, em dieta branda ou livre e que tinham ingerido pelo menos uma vez a refeição no almoço. Foram excluídos pacientes que se recusaram a participar da pesquisa, pacientes com deficiência de audição e visão e pacientes que apresentavam sintomas que impediam o andamento da entrevista como náuseas, vômitos, dor ou dificuldade em respirar.

Após a tabulação dos dados, foram determinadas ações para melhoria da qualidade do serviço de alimentação segundo o conceito de qualidade obtido como resultado do levantamento. A determinação das ações seguiu as seguintes etapas (MORIMOTO, 2002): 1) Diagnosticar a situação da característica selecionada; 2) Adequar ao conceito de qualidade do cliente; 3) Implementar a melhoria; 4) Avaliar a implementação da melhoria.

Para permitir comparação sobre a eficácia das ações, a chefia do SAN orientou a utilização de resultados de uma pesquisa de satisfação dos clientes internados com o serviço de alimentação, que havia sido realizada em novembro de 2018, anteriormente ao início deste estudo realizado que foi entre os meses de março a outubro de 2019. Os clientes de apartamentos e enfermarias analisaram os quesitos aparência dos alimentos, temperatura, quantidade, sabor, variação do cardápio e satisfação geral com as refeições atribuindo notas excelente, bom, regular, ruim, péssimo e não se aplica.

Para avaliar a implementação da melhoria, as mesmas pesquisas de satisfação e de aceitação da refeição foram repetidas, permitindo assim, a comparação entre a situação atual e anterior.

Para análise da satisfação, optou-se por agrupar as categorias excelente e bom denominando-as "satisfatórias" e regular, ruim e péssimo definindo-as como "não 
satisfatórias", além da categoria "não opinou". A análise descritiva foi realizada por meio de frequências, utilizando-se o programa Microsoft ${ }^{\circledR}$ Office Excel.

\section{RESULTADOS E DISCUSSÃO}

As etapas seguidas para determinação das ações estão apresentadas na Figura 1.

Figura 1. Fluxo de tomada de decisão para implementação de estratégias de melhorias em hospital oncológico. Curitiba, 2019.

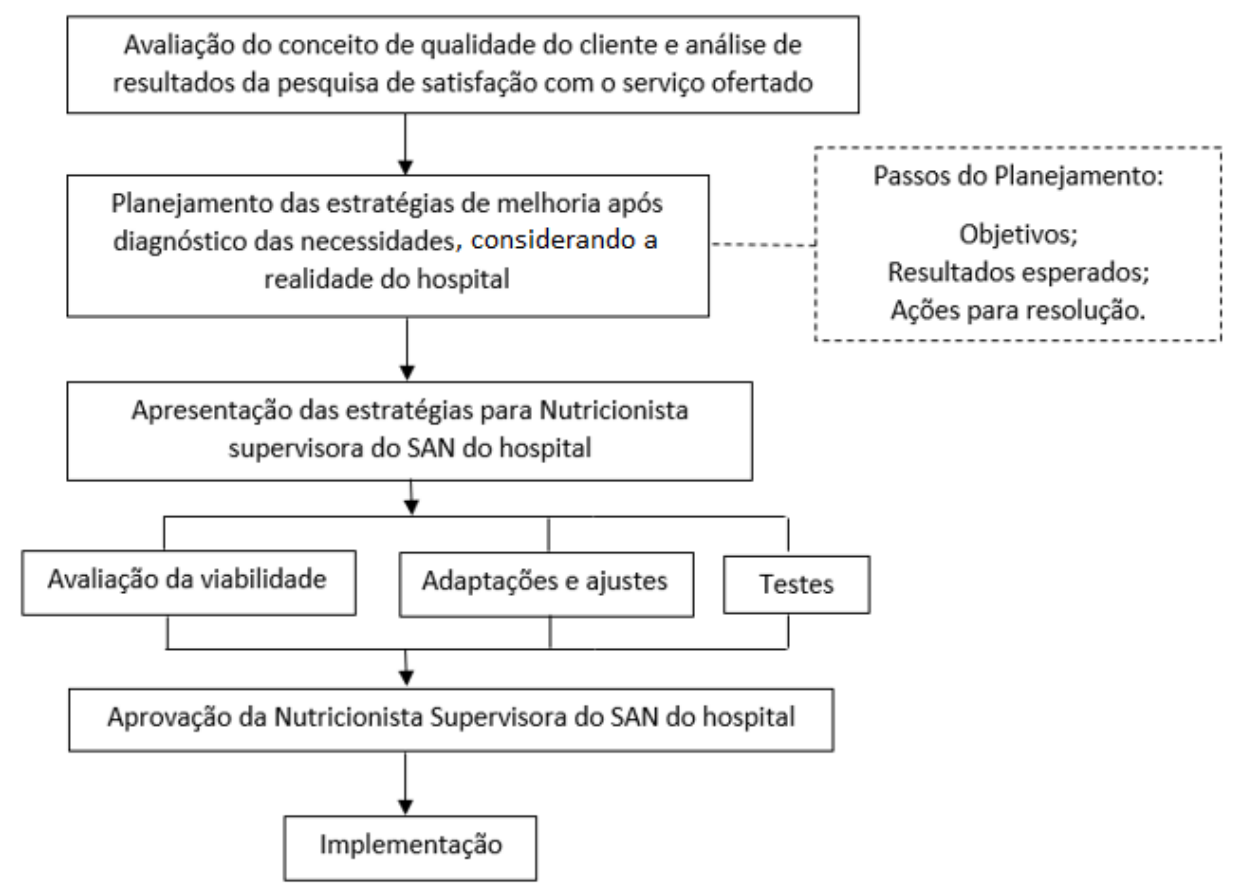

Para verificação dos determinantes da qualidade na visão do paciente oncológico hospitalizado, foram coletadas opiniões de 101 clientes sendo que 23 encontravam-se internados em apartamentos ( $83 \%$ do gênero feminino e $17 \%$ do gênero masculino) e 78 internados em enfermarias ( $64 \%$ homens e $36 \%$ mulheres).

Gráfico 1. Moda dos graus de importância das características de qualidade conferidas por clientes dos apartamentos e das enfermarias de hospital oncológico. Curitiba, 2019. 


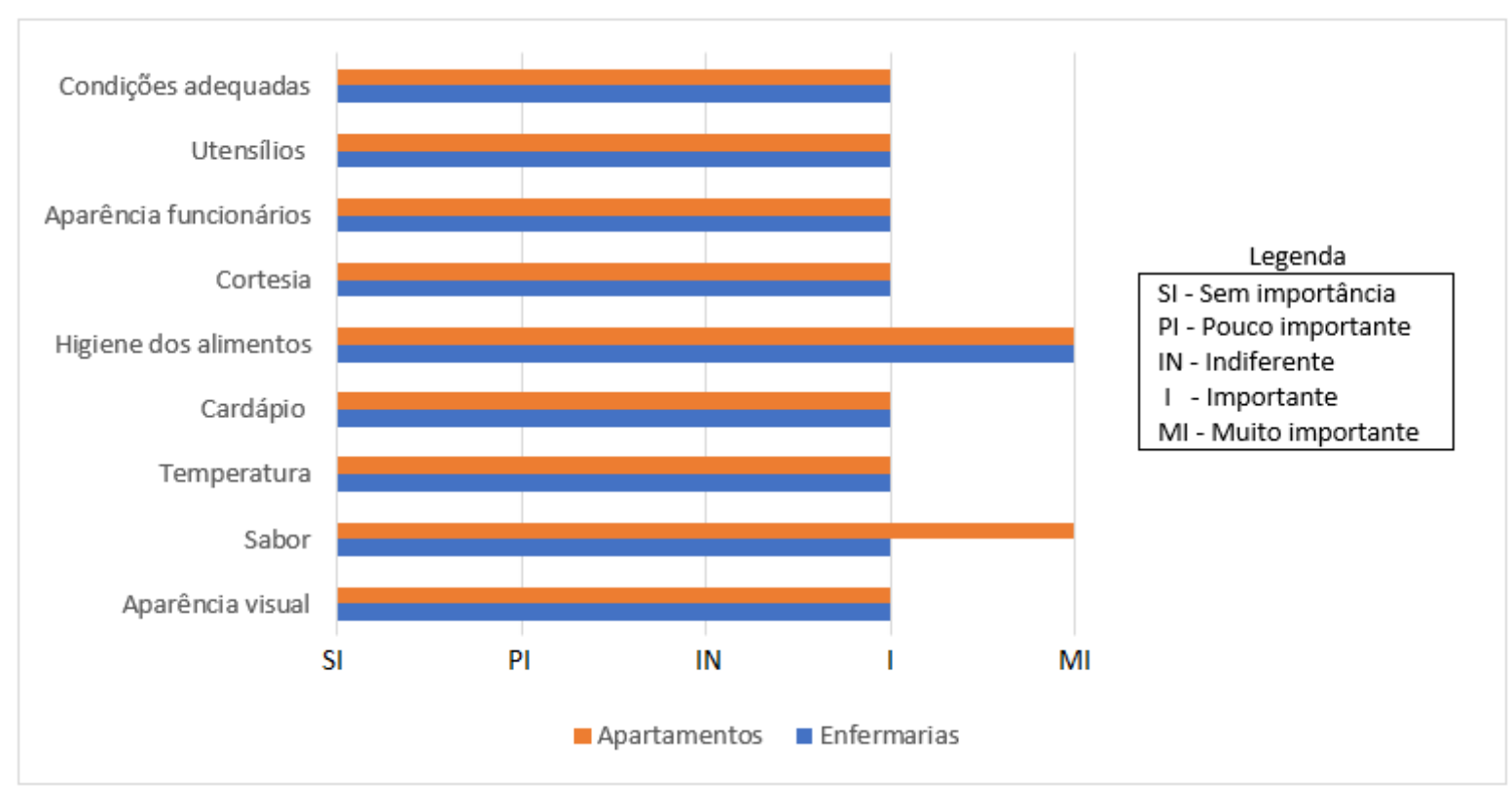

Fonte: As autoras, 2019.

\section{A Tabela 1 apresenta as estratégias discutidas e aprovadas pela nutricionista} supervisora do hospital com participação também de uma nutricionista clínica.

Tabela 1. Estratégias de melhoria para o serviço de alimentação em hospital oncológico com descrição da situação de implementação. Curitiba, 2019.

\begin{tabular}{|c|c|c|}
\hline $\begin{array}{l}\text { Características } \\
\text { do conceito de } \\
\text { qualidade }\end{array}$ & $\begin{array}{l}\text { Objetivo (O) } \\
\text { Resultado esperado (RE) }\end{array}$ & Ações Efetivadas (AE) e Ações Pendentes (AP) \\
\hline $\begin{array}{l}\text { Cortesia no } \\
\text { atendimento } \\
\text { de copeiras }\end{array}$ & $\begin{array}{l}\text { O: Melhorar a qualidade da } \\
\text { comunicação entre o SAN } \\
\text { hospitalar e clientes, de forma a } \\
\text { informar aos clientes os horários } \\
\text { das refeições, dos cardápios do dia } \\
\text { e alternativas de substituição de } \\
\text { refeições. } \\
\text { RE: Duas vias de comunicação } \\
\text { com o cliente: copeira e folder } \\
\text { informativo. }\end{array}$ & $\begin{array}{l}\text { AE: } \\
\text { a) Modificação do horário das refeições para atendimento à } \\
\text { pesquisa de satisfação: } \\
\text { - Modificação da rotina do serviço orientada pelo nutricionista } \\
\text { de produção. } \\
\text { b) Treinamento com copeiras do SAN: } \\
\text { - Sensibilização da copeira para empatia com o cliente e com } \\
\text { seu acompanhante e para clareza na informação; } \\
\text { - Orientação sobre informações a serem repassadas; } \\
\text { - Fluxo de encaminhamento das solicitações dos clientes para a } \\
\text { chefia. } \\
\text { c) Disponibilização de folder explicativo na porta de cada copa } \\
\text { das alas hospitalares contendo informações sobre os horários } \\
\text { das refeições, opções de pedidos (sopa ou refeição) e } \\
\text { especificações alimentares para cada ala para que copeiras, } \\
\text { enfermeiras e o próprio cliente tenham informações dos } \\
\text { motivos de horários de servimento das refeições. }\end{array}$ \\
\hline Higiene & $\begin{array}{l}\text { O: Garantir conhecimento do } \\
\text { Manual de Boas Práticas desde o } \\
\text { primeiro dia de serviço do } \\
\text { funcionário. } \\
\text { RE: Processos e fluxos corretos de } \\
\text { higiene pessoas, do ambiente e } \\
\text { alimentos. }\end{array}$ & $\begin{array}{l}\text { AE: } \\
\text { a) Elaboração de material educativo de Treinamento para Boas } \\
\text { Práticas na Manipulação de Alimentos. Material a ser } \\
\text { disponibilizado para novos colaboradores durante o programa } \\
\text { de integração do funcionário no hospital. }\end{array}$ \\
\hline Sabor & $\begin{array}{l}\text { O: Elaborar protocolo de } \\
\text { prevenção e tratamento de náuseas } \\
\text { e vômitos a partir de preparações } \\
\text { alimentares que combatem estes } \\
\text { sintomas. Além de aplicação de }\end{array}$ & $\begin{array}{l}\text { AE: } \\
\text { a) Seleção de receitas com critério base a utilização do gengibre } \\
\text { e de alimentos cozidos a fim de combater os sintomas de } \\
\text { náuseas e vômitos e atender o maior número possível de } \\
\text { clientes. }\end{array}$ \\
\hline
\end{tabular}

Revista Eletrônica Gestão e Serviços v.12, n. 1, pp. 3303 - 3318, Janeiro/Junho 2021.

ISSN Online: 2177-7284 e-mail: regs@metodista.br 
estratégias relacionadas a técnicas dietéticas no preparo dos alimentos para adequação da consistência.

RE: Combate aos sintomas comuns de náuseas e vômitos $\mathrm{e}$ adequação da consistência dos alimentos de acordo com as solicitações do cliente.

\begin{tabular}{ll}
\hline $\begin{array}{l}\text { Utensílios } \\
\text { adequados } \\
\text { para } \\
\text { alimentar-se }\end{array}$ & $\begin{array}{l}\text { O: Melhorar a qualidade dos } \\
\text { utensílios utilizados pelos clientes } \\
\text { do hospital. }\end{array}$ \\
& $\begin{array}{l}\text { RE: Alteração dos utensílios } \\
\text { utilizados no hospital por material } \\
\text { mais resistente e seguros aos } \\
\text { clientes. }\end{array}$ \\
$\begin{array}{l}\text { Condições } \\
\text { adequadas } \\
\text { para } \\
\text { alimentar-se }\end{array}$ & $\begin{array}{l}\text { apoio para o ato alimentar, } \\
\text { principalmente nas enfermarias. }\end{array}$ \\
& $\begin{array}{l}\text { RE: Fornecer bandejas e mobílias } \\
\text { adequadas para o ato alimentar dos } \\
\text { clientes, a fim de facilitar e } \\
\text { oferecer maior comodidade. }\end{array}$ \\
\hline
\end{tabular}

\begin{tabular}{ll}
\hline $\begin{array}{l}\text { Aparência } \\
\text { visual das } \\
\text { preparações }\end{array}$ & $\begin{array}{l}\text { O: Implementar conceitos e } \\
\text { práticas da gastronomia no } \\
\text { contexto de hotelaria hospitalar. }\end{array}$ \\
& $\begin{array}{l}\text { RE: Melhorar a aparência visual } \\
\text { das preparações desde o pré- } \\
\text { preparo até a montagem da }\end{array}$ \\
& $\begin{array}{l}\text { alimentação que é encaminhada } \\
\text { para o cliente. }\end{array}$
\end{tabular}

Legenda: SAN - serviço de alimentação e nutrição. Fonte: as autoras, 2019.
- Realização de testes e ajustes das receitas.

- Realização de teste sensorial completo feito pela equipe de nutricionistas do hospital;

- Elaboração de protocolo para aplicabilidade nos clientes, para melhora da aceitação alimentar através da redução desses sintomas gastrointestinais.

b) Aplicação de técnicas dietéticas para adequação da consistência de alguns alimentos de acordo com a solicitação do cliente:

- Acompanhamento do processo de produção de determinados alimentos que obtiveram comentários negativos em relação a consistência.

- Orientação feita as cozinheiras e dietistas referente ao tempo de cozimento e elaboração de estratégia a ser seguida para adequação da consistência.

AP

a) Pesquisas de modelos e orçamentos de utensílios;

- Identificação da necessidade de modificação dos talheres para de tipo mais resistente, de acordo com as solicitações dos clientes;

- Apresentação a supervisão do SAN, orçamentos de 3 tipos diferentes e de talheres não recicláveis de aço inoxidável;

\section{AP}

a) Pesquisas de modelos e orçamentos de utensílios;

- Identificação da necessidade de adequação de mobília de apoio para o ato alimentar, de acordo com as solicitações dos clientes;

- Apresentação a supervisão do SAN, orçamentos de 3 tipos diferentes de mobília, de bandeja de apoio;

- Encaminhamento do pedido ao setor de doações do hospital.
$\mathrm{AE}$

a) Treinamento destinado aos colaboradores do SAN (cozinheiros, auxiliares de cozinha e copeiras), com apoio de profissionais do Curso de Gastronomia:

- Demonstração de métodos de cortes para verduras e legumes;

- Utilização correta de facas e utensílios de cortes;

- Repetição das demonstrações de forma prática por todos os participantes do treinamento.

b) Treinamento com colaboradores do SAN (cozinheiros e copeiras):

- Elaboração de marmita piloto como exemplo para montagem das demais marmitas pelas copeiras, a fim de padronizar a apresentação das mesmas nas refeições de almoço e jantar, definindo a disposição dos alimentos e suas quantidades. Com base na apresentação da marmita piloto, as copeiras e dietistas realizaram a montagem das marmitas destinadas aos clientes

$\mathrm{Na}$ Tabela 2 estão discriminados os resultados da satisfação dos clientes com o SAN antes e depois da implementação das estratégias de melhoria. Houve aumento da frequência de respostas "satisfatório" relativas às características das refeições, atendimento das copeiras, horário de refeições e satisfação geral com o serviço que passou a ser ofertado.

Tabela 2. Comparação de grau de satisfação de clientes com dieta branda e livre antes e depois da implementação das estratégias no serviço de alimentação em hospital oncológico. Curitiba, 2019. 


\begin{tabular}{|c|c|c|c|c|c|c|}
\hline \multirow[b]{2}{*}{ CRITÉRIOS } & \multicolumn{3}{|c|}{$\operatorname{ANTES~}(n=62)$} & \multicolumn{3}{|c|}{ DEPOIS $(n=51)$} \\
\hline & $\begin{array}{l}\text { Satisfatório } \\
(\%)\end{array}$ & $\begin{array}{l}\text { Insatisfatorio } \\
(\%)\end{array}$ & $\begin{array}{l}\text { Não } \\
\text { opinou } \\
(\%) \\
\end{array}$ & $\begin{array}{l}\text { Satisfatório } \\
(\%)\end{array}$ & $\begin{array}{l}\text { Insatisfatorio } \\
(\%)\end{array}$ & $\begin{array}{l}\text { Não } \\
\text { opinou } \\
(\%)\end{array}$ \\
\hline \multicolumn{7}{|l|}{ REFEIÇÃO } \\
\hline Aparência da marmita & 80,66 & 17,73 & 1,61 & 92,16 & 7,84 & - \\
\hline $\begin{array}{l}\text { Satisfação geral com } \\
\text { relação ao almoço }\end{array}$ & 74,19 & 37,09 & 6,46 & 98,04 & 1,96 & - \\
\hline $\begin{array}{l}\text { Satisfação geral com } \\
\text { relação ao lanche da tarde }\end{array}$ & 74,2 & 12,89 & 12,9 & 90,2 & 1,96 & 7,84 \\
\hline $\begin{array}{l}\text { Satisfação geral com } \\
\text { relação ao jantar }\end{array}$ & 61,29 & 29,03 & 9,68 & 92,16 & 3,92 & 3.92 \\
\hline $\begin{array}{l}\text { Nível de satisfação geral } \\
\text { com as refeições }\end{array}$ & 79,03 & 17,74 & 3,23 & 96,08 & 3,92 & - \\
\hline \multicolumn{7}{|l|}{ COPEIRAS } \\
\hline Apresentação das copeiras & 91,94 & - & 8,06 & 100 & - & - \\
\hline $\begin{array}{l}\text { Cordialidade/gentileza das } \\
\text { copeiras }\end{array}$ & 93,55 & - & 6,45 & 100 & - & - \\
\hline $\begin{array}{l}\text { Clareza nas informações } \\
\text { dadas pelas copeiras }\end{array}$ & 91,94 & 1,61 & 6,45 & 100 & - & - \\
\hline $\begin{array}{l}\text { Agilidade no atendimento } \\
\text { das solicitações }\end{array}$ & 88,71 & 4,84 & 6,45 & 100 & - & - \\
\hline $\begin{array}{l}\text { Nível de satisfação geral } \\
\text { com as copeiras }\end{array}$ & 91,94 & 1,61 & 6,45 & 100 & - & - \\
\hline HORÁRIO & & & & & & \\
\hline $\begin{array}{l}\text { Horário do almoço } \\
\text { GERAL }\end{array}$ & 79,03 & 16,13 & 4,84 & 96,08 & 3,92 & - \\
\hline $\begin{array}{l}\text { Nível de satisfação geral } \\
\text { com o SAN }\end{array}$ & 91,94 & 6,45 & 1,61 & 100 & - & - \\
\hline
\end{tabular}

Fonte: As autoras.

Para verificar se as melhorias refletiram em maior aceitação da dieta oferecida, a Tabela 3 contém os dados da porcentagem de aceitação da dieta livre e branda pelos clientes de apartamentos e das enfermarias antes (realizada em 2018) e após (estudo de 2019) a implementação das melhorias. Observa-se que houve maior percentual de pacientes com aceitação de $75 \%, 50 \%$ e $25 \%$. Verificou-se também menor percentual de pacientes com $100 \%$ de aceitação da dieta.

Tabela 3. Comparação entre a aceitação do almoço de clientes com dieta branda e livre antes e depois da implementação das melhorias em hospital oncológico. Curitiba, 2019.

\begin{tabular}{lllll}
\hline \multirow{2}{*}{$\begin{array}{l}\text { Percentual } \\
\text { de aceitação }\end{array}$} & \multicolumn{2}{c}{ ANTES $(\mathbf{n = 1 0 1})$} & \multicolumn{2}{c}{ DEPOIS (n=51) } \\
\cline { 2 - 5 } $\mathbf{\mathbf { n } ^ { \mathbf { 0 } }}$ & $\mathbf{\%}$ & $\mathbf{n}^{\mathbf{0}}$ & $\mathbf{\%}$ \\
\hline $0 \%$ & 6 & 5,94 & 2 & 3,92 \\
$25 \%$ & 13 & 12,9 & 11 & 21,56 \\
$50 \%$ & 16 & 15,84 & 11 & 21,56 \\
$75 \%$ & 26 & 25,74 & 11 & 21,56 \\
$100 \%$ & 40 & 39,6 & 16 & 31,4 \\
\hline Fonte: as autoras, 2019. & & &
\end{tabular}

A Tabela 4 apresenta a comparação dos motivos da não-aceitação alimentar nos casos de consumo inferior a $100 \%$. Dentre estes motivos, estão os relacionados ao serviço prestado tanto pelo pessoal do SAN, responsável pelo porcionamento e preparo 
dos alimentos quanto dos nutricionistas clínicos, responsáveis pela adequação da consistência para clientes hospitalizados com problemas de mastigação.

Os motivos dependentes do serviço incluem as características organolépticas, a quantidade exagerada dos alimentos do ponto de vista dos clientes, a consistência inadequada e a oferta de alimentos que não correspondem à preferência. Nestes quesitos, houve redução de frequência de $26,26 \%$ para 20,51 .

Dentre os motivos de não aceitação relacionados a doença, foi possível identificar a falta de apetite e presença de distúrbios gastrointestinais nos dois momentos, porém, com aumento de $73,73 \%$ para $79,29 \%$ depois, confirmando que a não aceitação recebeu grande influência de fatores relacionados à doença.

Ressalta-se que foram relatados mais de um motivo de não aceitação por cliente internado, tanto antes quanto depois da implementação das estratégias.

Tabela 4. Comparação entre motivos da não aceitação alimentar em hospital oncológico. Curitiba, 2019.

\begin{tabular}{|c|c|c|c|c|c|}
\hline \multicolumn{2}{|c|}{ Motivos de não aceitação da dieta } & \multicolumn{2}{|c|}{ ANTES (n=99) } & \multicolumn{2}{|c|}{ DEPOIS (n=73) } \\
\hline & & $\mathbf{n}^{0}$ & $\%$ & $\mathbf{n}$ & $\%$ \\
\hline \multirow{4}{*}{$\begin{array}{l}\text { Dependentes do } \\
\text { serviço prestado }\end{array}$} & Características organolépticas & 6 & 6,06 & 3 & 4,1 \\
\hline & Quantidade exagerada & 9 & 9,09 & 2 & 2,73 \\
\hline & Consistência inadequada & 4 & 4,04 & 4 & 5,47 \\
\hline & $\begin{array}{l}\text { Não corresponde às preferências } \\
\text { alimentares }\end{array}$ & 7 & 7,07 & 6 & 8,21 \\
\hline \multirow{6}{*}{$\begin{array}{l}\text { Relacionados à } \\
\text { doença }\end{array}$} & Falta de apetite & 34 & 34,34 & 23 & 31,5 \\
\hline & Distúrbios gastrointestinais & 16 & 16,16 & 14 & 19,17 \\
\hline & Dificuldade de mastigação & 9 & 9,09 & 9 & 12,32 \\
\hline & Dificuldade de deglutição & 7 & 7,07 & 9 & 12,32 \\
\hline & Dor ao deglutir & 3 & 3,03 & 3 & 4,1 \\
\hline & Distúrbios psicológicos & 4 & 4,04 & 0 & 0 \\
\hline
\end{tabular}

Fonte: As autoras, 2019.

\section{DISCUSSÃO}

A Norma Técnica da ABNT NBR ISO 9001:2015 refere-se a requisitos para um sistema de gestão da qualidade para as organizações. Nessa norma, quando se trata de avaliação de desempenho, descrevem-se as etapas de monitoramento, medição, análise e avaliação. Além disso, destaca que é necessário um sistema de gestão da qualidade que monitore as percepções dos clientes, levando em consideração o atendimento de suas necessidades e expectativas (QUEIROZ, 2016). Juran (1992) afirma que isso deve ser garantido por meio do estabelecimento de uma infraestrutura de feedback dos clientes que conduzam a novos projetos de melhoramento, o que denominou de processo de melhoria contínua. As pesquisas de satisfação podem ser utilizadas como ferramentas 
para identificar problemas e aprimorar estratégias de mudanças, a fim de atingir o conceito de qualidade do cliente hospitalizado (QUEIROZ, 2016).

Neste estudo, para cada inadequação identificada no serviço relacionada aos fatores determinantes da qualidade na visão do foram estabelecidos objetivos a serem alcançados, os resultados esperados e as ações a serem tomadas para resolução da inadequação, seguindo o Modelo de Melhoria da Qualidade em Serviços de Alimentação Hospitalar (MORIMOTO, 2002). As etapas de melhoria on-line são fundamentadas no processo produtivo - otimização de atividades e minimização de custos, considerando as potencialidades que agregam valor ao produto (PALADINI, 1995). Neste aspecto, as ações objetivaram a melhoria da aparência e garantia da higiene das refeições servidas e foram compostas de: material de treinamento para cozinheiras, dietistas e auxiliares de cozinha sobre boas práticas na manipulação dos alimentos; treinamento com aplicação de práticas gastronômicas no contexto de hotelaria hospitalar; treinamento com copeiras para aplicação da marmita piloto no serviço.

A estratégia de melhoria de cortes dos alimentos e ações como a marmita piloto, considera conceitos da gastronomia no contexto de hotelaria hospitalar. De acordo com Calza (2017), novos conceitos de gastronomia hospitalar, melhoram as caracteristicas sensoriais da comida, a apresentação das dietas e o atendimento ao cliente podendo auxiliar na prevenção ou reversão da desnutrição em pacientes oncológicos.

Uma característica da qualidade muito valorizada pelo cliente hospitalizado é a higiene. A internação gera sentimento de insegurança e fragilidade, e a partir disto, o cliente busca referências de segurança e os alimentos se tornam inseridos no conceito de qualidade higiênica e de segurança alimentar para o cliente (MORIMOTO; PALADINI, 2009). A RDC no 216, de 15 de setembro de 2004, determina o estabelecimento de “procedimentos de Boas Práticas para serviços de alimentação a fim de garantir as condições higiênico- sanitárias do alimento preparado." (BRASIL, 2004). Por isso destaca-se a importância da capacitação de profissionais que foi realizada com base no Manual de Boas Práticas do serviço, favorecido pela adequada estrutura física do SAN inaugurado em dezembro de 2018, anterior ao início do projeto. No trabalho de Shinohara et al. (2016), foi destacado também que critérios de higiene e o conhecimento sobre o Manual de Boas Práticas do estabelecimento devem ser abordados durante a 
capacitação dos colaboradores que atuam na manipulação dos alimentos a fim de garantir a segurança alimentar do SAN.

As estratégias on-line que têm relação direta com a satisfação dos clientes, são estratégias relativas à preferências do consumidor (MORIMOTO, 2002). Foram realizadas no sentido de conferir conforto para o ato de alimentar-se, gerar práticas de atitudes de cortesia no atendimento e melhorar a comunicação referente à horários das refeições. A comida de hospital, concebida como insossa, sem gosto, fria, servida muito antes do horário de rotina e ainda com conotações de permissão e proibição está arraigada na cultura popular (DEMÁRIO; SOUZA; SALLES, 2009; SOUZA; PROENÇA, 2005). O paciente hospitalizado necessita muitas vezes de modificações na dieta que limitam a quantidade de sal, açúcar, pimenta, bem como a textura para alimentos mais macios que interferem no paladar. Neste momento, os nutricionistas clínicos que compõem o primeiro time de contato do serviço de nutrição com o paciente hospitalizado atuam explicando aos pacientes sobre a necessidade de adequação da alimentação de maneira a permitir a atuação da dietoterapia como auxiliar no tratamento. Preparações alimentares específicas com gengibre para diminuir o sintoma de náuseas, que é um dos sintomas mais prevalentes da não aceitação alimentar em pacientes oncológicos também foram executadas. (SOUZA et al., 2019). Estes “sintomas decorrentes da própria doença e de seus tratamentos possuem importante relação com a ingestão alimentar e o consequente estado nutricional do paciente", (PALMIERI et al., 2013).

Segundo Parasuramam, Zeithaml, Berry (1998), e Iribarrem (2006) elaborar uma estratégia para melhorar a percepção da comunicação entre paciente e SAN hospitalar é fundamental para se obter a qualidade no serviço. Neste estudo, houve ajustes de horários da refeição do almoço, para mais cedo, conforme demandas dos clientes. Além desta ação, durante o treinamento de comunicação e cortesia no atendimento com as copeiras foi orientada a comunicação com o cliente referente aos horários das refeições. Não houve possibilidade de avaliação do conforto para o ato da alimentação até a finalização do estudo por fatores orçamentários. "Os móveis hospitalares de qualidade contribuem para o bem-estar do cliente e de seu acompanhante, não estando ligados ao luxo, mas sim ao conforto, segurança e boa condição na prestação de serviço", (NÓBREGA, 2017), atuando também na melhoria da aceitação alimentar. 
Após implementação das estratégias observou-se aumento da satisfação com o serviço prestado. No primeiro momento (novembro de 2018) havia percepção do serviço como insatisfatório por parte de alguns pacientes e no segundo (outubro de 2019) as opiniões apresentadas foram somente de satisfatório, que incluíram as categorias excelente e bom. Schmidt, et al. (2014) realizou uma pesquisa de satisfação referente ao SAN de um hospital universitário, utilizando escala Likert de 5 pontos, de totalmente insatisfeitos com o serviço ofertado à totalmente satisfeitos. A pesquisa obteve uma avaliação satisfatória quanto a qualidade da alimentação e adequação dos horários para alimentação, assim como também foi obtido na presente pesquisa. porém, não apresentam informações sobre implementação de estratégias de melhorias.

No estudo de Pfaffenzeller, (2003) em ambiente hospitalar os resultados da pesquisa de satisfação, utilizando a escala Likert serviram como base para a elaboração de um plano de intervenção que incluiu os seguintes aspectos: ação, procedimento, objetivo, responsável, local e data/período. Este plano de intervenção incluiu itens semelhantes ao proposto por este estudo, reforçando a necessidade de sistematização de dados e de utilização de metodologias para determinação de estratégias visando a qualidade do serviço prestado ao cliente hospitalizado.

Embora os autores afirmem que a viabilidade de aplicar o conceito do cliente ao serviço, de modo a individualizar as preferências e satisfazer os clientes, resulta em benefício a melhoria da aceitação das dietas hospitalares (SOUZA; SALLES et al., 2013), isso não ocorreu neste estudo. Destaca-se que, a aceitação da dieta hospitalar nunca chegará a $100 \%$ devido à presença constante e inevitável de sintomas relacionados a doença, as cirurgias, a quimioterapia e radioterapia. Segundo Ferreira, Guimarães, Marcadenti (2013), entre pacientes hospitalizados com câncer, o resto ingestão indica um elevado índice (37\%) quando comparado com outras populações enfermas (20\%). O principal motivo da não aceitação foram os sintomas de náusea e vômito. Estes sintomas em clientes com câncer estão relacionados com a própria neoplasia ou toxicidade do tratamento. Clientes em tratamento quimioterápico tem a náusea e o vômito como efeito adverso, acometendo até $80 \%$ dos clientes sob tratamento (BARRETO; TOSCANO; FORTES, 2012).

No caso dos pacientes deste estudo, fatores relacionados à própria doença ou seu tratamento, resultaram em resultados negativos sobre a aceitação. Apesar disso, houve sinais positivos quando verificados fatores dependentes do serviço de alimentação como 
porcionamento de quantidade exagerada de alimentos e características organolépticas da refeição, denotando eficácia dos procedimentos adotados. Algumas estratégias envolvendo investimento financeiro como aquisição de mobílias, bandejas e talheres, não puderam ser implantadas até a finalização deste estudo pois envolviam outras instâncias administrativas impossibilitando a verificação dos resultados referentes ao conforto para a alimentação.

\section{CONCLUSÃO}

A avaliação de resultados de estratégias para melhoria da qualidade do SAN de um hospital oncológico a partir dos fatores determinantes da qualidade na visão deste cliente permitiu verificar particularidades quando se trata do câncer. A quimioterapia, radioterapia e o tratamento cirúrgico a que o cliente é submetido durante a internação resultam em sintomas que interferem negativamente na aceitação alimentar. Entretanto, isso não invalida a necessidade de realizar o levantamento do conceito de qualidade do cliente em pesquisas periódicas e que esses determinantes da qualidade sejam considerados na elaboração de estratégias seguindo metodologia que envolve objetivos, resultados esperados e ações efetivas. Ressalta-se que caso a satisfação com fatores dependentes do serviço como sabor, higiene, cortesia no atendimento e cardápio não sejam atendidos, a aceitação alimentar pode reduzir ainda mais, prejudicando desta maneira o tratamento do cliente oncológico hospitalizado.

Existem no hospital protocolos de nutrição para cada fator de redução da aceitação e neste aspecto, destaca-se o papel essencial do nutricionista clínico, juntamente com a equipe multidisciplinar no diagnóstico e intervenção diante destes sintomas, para viabilizar maior ingestão alimentar.

Observou-se carência de estudos relacionados a resultados de implementação de estratégias de melhoria da qualidade em serviços de alimentação e nutrição hospitalares, portanto, sugere-se mais estudos nesta área.

\section{REFERÊNCIAS}

AQUINO, R. C.; PHILIPPI, S. T. Identificação de fatores de risco de desnutrição em pacientes internados. Revista da Associação Médica Brasileira. São Paulo, v. 57, n. 6, p. 637-643, ago., 2011. 
BANKS, M. et al. Measuring the quality of hospital food services:development and reliability of a meal qualityaudit tool. Nutrition\&dietetics, Austrália, v. 74, n. 2, p. 147-157, 2017.

BARRETO, A. M. C.; TOSCANO, B. A. F.; FORTES, R. C. Efeitos do gengibre (Zingiber officinale) em pacientes oncológicos tratados com quimioterapia.

Comunicação em Ciências da Saúde. Brasília, v. 22, n. 3, p. 257 - 270, mar., 2012.

Disponível em:

$<\mathrm{http} / /$ http://bvsms.saude.gov.br/bvs/periodicos/revista_ESCS_v22_n3_a08_efeitos_ge ngibre.pdf $>$. Acesso em: 15 out. 2019.

BONATO, V. L. Gestão de qualidade em saúde: melhorando assistência ao cliente. O Mundo da Saúde. Biblioteca Virtual em Saúde - Ministério da Saúde, São Paulo, v. 35, n.5, p. 319-331, 2011.

BRASIL. Ministério da Saúde. Agência Nacional de Vigilância Sanitária. Resolução: RDC No 216, de 15 de setembro de 2004. Brasília, 2004. 14 p. Disponível em: $<\mathrm{http} / /$ http://portal.metodista.br/biblioteca/servicos/manual-de-referencias-2.htm>. Acesso em 20 set. 2019.

CALZA, G. F. Gastronomia hospitalar e aceitação de dietas em pacientes oncológicos pediátricos. 2017. 35p. Tese (Pós Graduação em Gestão da Produção de Refeições Saudáveis) Universidade de Brasília, Brasília/BR, 2017.

CARVALHO, G.; CAMILO, M. E.; RAVASCO, P. Qual a relevância da nutrição em oncologia? Acta Médica Portuguesa. Lisboa, v. 24, n. S4, p. 1041-1050, 2011.

Disponível em: $<$ http://

https://www.actamedicaportuguesa.com/revista/index.php/amp/article/viewFile/1561/11 45>. Acesso em: 17 out.2019.

CARPINETTI, L. C. R. Gestão da Qualidade: conceitos e técnicas. 2a edição. São Paulo, Atlas, p. 256, 2012.

DEMÁRIO, R. L.; SOUZA, A. A.; SALLES, R. K. Comida de hospital: percepções de pacientes em um hospital público como proposta de atendimento humanizado. Ciência \& Saúde Coletiva. Florianópolis, v. 15, n. 1, p. 1275-1282, 2009.

FERREIRA, D.; GUIMARÃES, T. G.; MARCADENTI, A. Aceitação de dietas hospitalares e estado nutricional entre pacientes com câncer. Journal Einstein. Porto Alegre, v. 11, n. 1, p. 41-46, 2013. Disponível em: < https://journal.einstein.br/wpcontent/uploads/articles_xml/1679-4508-eins-S1679-45082013000100008/1679-4508eins-S1679-45082013000100008.x23626.pdf> . Acesso em: 30 jun.2019.

FIGUEIREDO, T. et al. Como posso ajudar? Sentimentos e experiências do familiar cuidador de pacientes oncológicos. ABCS Health Sciences. São João del-Rei, v. 42, n. 1, p. 34-39, 2017. Disponível em:

$<$ https://portalnepas.org.br/abcshs/article/viewFile/947/759>. Acesso em: 01 out.2019.

IRIBARREM, K.H. Estudo e melhoria dos serviços de alimentação do hospital moinhos de vento - Porto Alegre/RS. 2006. 110f. Dissertação (Mestrado em Engenharia de Produção) - Faculdade de Engenharia da Universidade Federal do Rio Grande do Sul, Porto Alegre, 2006.

JURAN, J. M. A qualidade desde o projeto: novos passos para o planejamento da qualidade em produtos e serviços. $2^{\mathrm{a}}$ edição. São Paulo: Pioneira, p. 551, 1992. 
MORIMOTO, I. I. M. Melhoria da qualidade na unidade de alimentação e nutrição hospitalar: um modelo prático. 2002. 261f. Dissertação (Mestrado em Engenharia de Produção) - Faculdade de Engenharia de Produção da Universidade Federal de Santa Catarina, Florianópolis, 2002.

MORIMOTO, I. I. M.; PALADINI, E. P. Determinantes da qualidade da alimentação na visão de pacientes hospitalizados. O Mundo da Saúde, São Paulo- SP. v.33, n.3, p. 329-334, 2009. Disponível em: $<\underline{\text { http://www.saocamilo- }}$ sp.br/pdf/mundo_saude/69/329a334.pdf $>$. Acesso em 02 set.2019.

NÓBREGA, L. R. Avaliação e comparação do mobiliário hospitalar de diferentes unidades de internação. 2017. 150p. Dissertação (Mestrado em Engenharia Biomédica) Faculdade de Engenharia Elétrica da Universidade Federal de Uberlândia, Uberlândia, 2017.

PALADINI. E, Gestão da qualidade no processo. São Paulo: Atlas, 1995.

PALMIERI, B. N. et al. Aceitação de preparações e sua associação com os sintomas decorrentes do tratamento de câncer em pacientes de uma clínica especializada.

Cadernos Saúde Coletiva. Rio de Janeiro, v. 21, n. 1, p. 2-9, 2013.

PARASURAMAN, A.; ZEITHAML, V.O.; BERRY, L. Servqual: a multiple - item sacale for mensuring consumer perceptions of servisse quality. Journal of Retailing. Texas, v. 64, n. 1, p. 1-29, 1998. Disponível em: $<$ https://www.researchgate.net/profile/Valarie Zeithaml/publication/225083802_SE RVQUAL_A_multiple_Item_Scale_for_measuring_consumer_perceptions_of_service quality/links/5429a4540cf27e39fa8e6531/SERVQUAL-A-multiple-Item-Scale-formeasuring-consumer-perceptions-of-service-quality.pdf $>$. Acesso em: 25 ago.2019.

PFAFFENZELLER, A. A. Assistência nutricional hospitalar: um estudo da satisfação dos clientes da Santa Casa de Porto Alegre. 2003. 139p. Dissertação (Mestrado Profissionalizante em Engenharia) Universidade Federal do Rio Grande do Sul, Porto Alegre, 2003.

QUEIROZ, P. S. C. A importância do monitoramento para a qualidade de serviços: estudo de caso em Academia de Ginástica Feminina na cidade de João Pessoa PB. 2016. 174f. Tese (Especialização em Administração) Instituto Federal de Educação, Ciência e Tecnologia, João Pessoa, 2016.

RIBAS, S. A. et al. Determinantes do grau de aceitabilidade da dieta hospitalar: ferramentas para a prática clínica? Demetra Alimentação, Nutrição \& Saúde. Rio de Janeiro, v. 8, n. 2, p. 137-148, 2013. Disponível em: $<$ https://www.epublicacoes.uerj.br/index.php/demetra/article/view/3788/5142>. Acesso em: 17 out. 2019.

ROMERO, G.; ANGST, C. A. Serviço de Alimentação e Nutrição Hospitalar: elaboração de fichas técnicas. Disciplinarum Scientia. Santa Maria, v. 16, n. 2, p. 265273, 2015. Disponível em:

https://periodicos.ufn.edu.br/index.php/disciplinarumS/article/view/1017/961> Acesso em: 17 out. 2019.

SAIATO, D. Y. T. et al. Usuário, cliente ou paciente? Qual termo mais utilizado pelos estudantes de enfermagem? Texto Contexto Enfermagem. Florianópolis, v.22, n.1, p. 175-83, 2013. 
SALCI, M. A.; MARCON, S. S. A convivência com o fantasma do câncer. Revista Gaúcha Enfermagem. Porto Alegre, v. 31, n. 1, p. 18-25, 2010.

SCHMIDT, S. M. S. et al.; Análise da satisfação dos usuários em um hospital universitário. Saúde Debate. Rio de Janeiro, v. 38, n. 101, 2014. Disponível em:< https://www.scielo.br/pdf/sdeb/v38n101/0103-1104-sdeb-38-101-0305.pdf > Acesso em: 21 dez. 2020.

SHINOHARA, N. K. S. et al. Boas práticas em serviços de alimentação. Revista Eletrônica Diálogos Acadêmicos. Pernambuco, v.10, n.1, p. 79-91, 2016. Disponível em: <http://uniesp.edu.br/sites/_biblioteca/revistas/20170627113500.pdf $>$ Acesso em: 21 dez. 2020.

SOUZA, A. A.; PROENÇA, R. P. C. La gestion des soins nutritionnels dans le secteur horpitalier: une etude comparative Bresil- France. Rech Soins Infirm. Florianópolis, v. 83, p. 28- 33, 2005. Disponível em: $<$ https://nuppre.ufsc.br/files/2014/04/2005-SOUSAet-al.pdf>. Acesso em: 15 out. 2019.

SOUZA, A. A.; SALLES, R. K. et al. Alimentação hospitalar: elementos para a construção de iniciativas humanizadoras. Demetra: Alimentação, Nutrição \& Saúde. Florianópolis, v.8, n.2, p. 149-162, 2013. Disponível em: $<$ https://www.epublicacoes.uerj.br/index.php/demetra/article/view/5281/5143>. Acesso em: 15 out. 2019.

SOUZA, J. P. et al. Breve relato sobre os efeitos terapêuticos do gengibre (Zengiber officinale Roscoe). Revista Científica da Faculdade de Educação e Meio Ambiente. Ariquemes- RO. v.10, n.1, p. 44-53, 2019. Disponível em:< http://www.faema.edu.br/revistas/index.php/Revista-FAEMA/article/view/785>. Acesso em: 13 ago.2019.

TRANCOSO, S. C.; TOMASIAK, F. S. Estruturação de uma unidade de alimentação e nutrição. Nutrição Brasil. Rio de Janeiro, v. 3, n. 1, p. 12, fev., 2004.

VIEIRA, R. M. et al. Avaliação qualitativa das preparações oferecidas em um serviço de nutrição e dietética hospitalar. Revista Uniabeu. Guarapuava- PR. v.9, n.23, p. 151161, 2016. Disponível em:< https://revista.uniabeu.edu.br/index.php/RU/article/view/2478/pdf $>$. Acesso em: 13 jul.2019. 